\title{
Nachwirkungserscheinungen am keramischen Bariumtitanat
}

\author{
Bertram Wild \\ Institut für Theoretische Physik der Technischen Hochschule Karlsruhe \\ (Z. Naturforschg. 21 a, 1672-1683 [1966] ; eingegangen am 4. April 1966)
}

\begin{abstract}
An grobkristallinem Bariumtitanat wurden im Temperaturbereich 10 bis $110^{\circ} \mathrm{C}$ und im Zeitintervall von 2 bis $10^{7} \mathrm{sec}$ Nachwirkungsströme gemessen, ebenso Ausheizströme bis zu Temperaturen über $600{ }^{\circ} \mathrm{C}$. Dabei ergaben sich drei Relaxationsmechanismen: der rein ferroelektrische, ein von den normalen Randschichten der Kristallite verursachter und schließlich eine Nachwirkung auf Grund der Randschichten, die durch einseitige Diffusion von Elektrodenatomen entstehen.
\end{abstract}

Bei der Untersuchung keramischer Bariumtitanatproben $\left(\mathrm{BaTiO}_{3}\right)$ fanden verschiedene Autoren Zeiteffekte. $\mathrm{KocH}^{1}{ }^{3}$ untersuchte als erster systematisch die Änderung der Dielektrizitätskonstanten (DK) nach plötzlichem Einschalten bzw. Abschalten eines elektrischen Gleichfeldes. Zur qualitativen Deutung seiner Messungen zog er zwei Effekte heran, 1. die Abnahme der DK durch das Wachsen energetisch günstig orientierter Bereiche und 2. ihre Zunahme durch neue (bzw. alte, beweglicher gewordene) Wände. Weitere Messungen von $\mathrm{W}_{\text {AGNER }}{ }^{2 \mathrm{a}-\mathrm{c}}$ über den Einfluß der Anfangs-DK auf den Einschaltanstieg kann man ebenfalls auf Grund dieser Vorstellungen verstehen.

Die Nachwirkungserscheinungen nur durch Messung der DK zu verfolgen, hat Nachteile: 1. Die Wechselspannung zur Messung der DK verfälscht bei kleinen Gleichfeldern das Meßergebnis. 2. Die interessierenden DK-Änderungen betragen nur wenige Prozent und werden daher leicht durch Störeffekte beeinflußt.

Daher lag es nahe, eine einfacher meßbare Größe auszuwählen, die außerdem nach Möglichkeit enger mit den eigentlich interessierenden Größen, der dielektrischen Verschiebung bzw. Polarisation, zusammenhängt. Eine solche ist zunächst der Strom, der nach dem Anlegen bzw. Abschalten des elektrischen Gleichfeldes fließt. Er kann ohne zusätzliche Meßfelder bestimmt werden. Beim „Laden“ stört zwar der zusätzliche Leitfähigkeitsstrom, dieser fällt jedoch beim „Entladen“ weg. Da der Entladestrom andererseits im Prinzip unendlich lang fließt und da-

1 W. Косн, Z. Naturforschg. 13 a, 303 [1958], s. dort auch weitere Literaturangaben. Die Arbeit J. Gürrci u. a. (Phys. Status Solidi 6, 174 [1964]) braucht nur erwähnt zu werden, da sie bei weitem nicht die Ergebnisse von Косн erreicht.

2a R. WAGNER, Diplomarbeit 1960, Institut für theoretische Physik der Technischen Hochschule Karlsruhe. bei schließlich unmeßbar klein wird, ist es zweckmäßig, diesen Vorgang durch Hochheizen der Probe zu beschleunigen. Die vorliegende Arbeit befaßt sich daher hauptsächlich mit dem Entladestrom und dem Ausheizstrom. Besonders dieser letzte hilft, in eine Vielfalt von Erscheinungen Ordnung zu bringen (vgl. die Übersicht am Schluß, Abschnitt 8).

\section{Kurze Theorie der elektrischen Nachwirkung}

Wenn man die elektrische Feldstärke in einem Medium - etwa durch Anlegen einer Gleichspannung an einen Kondensator - plötzlich ändert, so folgen in der Gleichung $\mathfrak{D}=\varepsilon_{0} \mathfrak{F}+\mathfrak{A}$ die Größen $\mathfrak{D}$ und $\mathfrak{\Re}$ nicht sofort, sondern mit einer gewissen Verzögerung, d. h. außer der Ladung $\varepsilon_{0} E F_{\text {Elektrode }}$, die (unter der Annahme, daß der Zuleitungswiderstand Null ist) sofort beim Ändern von E auf die Kondensatorplatten strömt, fließt auch nachher noch ein Strom, der Nachwirkungsstrom $J_{\mathrm{N}}=F_{\text {Elektrode }} j_{\mathrm{N}}$, wobei

$$
j_{\mathrm{N}}=-\partial \mathfrak{D} / \partial t=-\partial \mathfrak{P} / \partial t .
$$

Dazu kommt bei $E \neq 0$ noch die Leitfähigkeitsstromdichte $j_{\mathrm{L}}=\sigma \mathfrak{E}$. Für den zeitlichen Verlauf von $\mathfrak{D}$ nach einer sprunghaften Änderung von $\leftleftarrows$ sind viele Annahmen möglich. Wir machen hier deshalb rein formal - ohne ein spezielles Modell vorauszusetzen - wie schon oft verwandt für den Betrag von $\mathfrak{D}$, einen Exponentialansatz

$$
D=D_{0} e^{-t / \tau},
$$

wobei $\tau$ die Relaxationszeit ist.

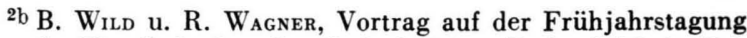
der Physikalischen Gesellschaft Württ.-Baden-Pfalz in Heidelberg 1960; Phys. Verh. 11, 80 [1960].

${ }^{2 c}$ B. WILD, Vortrag auf der Frühjahrstagung der Physikalischen Gesellschaft Württ.-Baden-Pfalz in Ulm 1959, Phys. Verh. 10, 10 [1959]. 
Nach dem Verhalten von $D_{0}$ kann man zwei gegensätzliche Modelle unterscheiden. Das eine wird besonders einfach durch die Hintereinanderschaltung von Widerstand und Kapazität dargestellt. Bei diesem „umkehrbaren“ Relaxationsmechanismus beschreibt das gleiche $\tau$ den Lade- und den Entladevorgang. Die Konstante $D_{0}$ ist beim Ladevorgang nur durch das Produkt von Ladespannung und Kapazität bestimmt. Beim Entladevorgang dagegen hängt $D_{0}$ außerdem noch von der Ladezeit $\Delta_{1}$ ab. $D_{0}$ wächst bei sonst unveränderten Umständen - mit $\Delta_{1}$ gemäß

$$
D_{0}=D_{0, \max }\left[1-\exp \left(-\Delta_{1} / \tau\right)\right],
$$

d. h. soll $D_{0}$ weniger oder höchstens um $1 \%$ von $D_{0, \max }$ abweichen, so muß $\Delta_{1} \geqq 4,6 \tau$ sein. - Das andere Modell ( „nicht umkehrbarer" Nachwirkungsmechanismus) wird im Extremfall verwirklicht durch das Überbrücken des Widerstandes beim Ladevorgang, d. h. die Aufladung erfolgt sofort. Bei der Entladung dagegen soll der Widerstand nicht überbrückt sein, und wir finden einen exponentiellen Nachwirkungsstrom, dessen Konstante $D_{0}$ nicht von der Ladezeit abhängt.

Eine einzelne Exponentialfunktion reicht jedoch zur Beschreibung allgemeinerer Fälle nicht aus. Man erweitert daher den Ansatz in zwei Schritten:

Zuerst nimmt man eine Verteilung verschiedener Relaxationszeiten $\tau$ um einen häufigsten Wert $\tau_{\max }$ gemäß der Gauss-Funktion $\exp \left[-(z / b)^{2}\right]$ an, wobei $z=\ln \left(\tau / \tau_{\max }\right)$ und $b$ ein Maß für die Breite der Gauss-Funktion und damit für die Streuung der Relaxationszeiten ist. Mit dieser Annahme erhält man für die normierte Nachwirkungsstromdichte $j_{\text {normiert }}$ die von $\mathrm{W}_{\mathrm{AGNER}}{ }^{3}$ eingeführte Nachwirkungsfunktion

$$
\begin{aligned}
\eta(t) & \equiv j_{\text {normiert }}=\frac{j_{\mathrm{N}} b \sqrt{\pi} \tau_{\max }}{D_{0}} \\
& =\int_{-\infty}^{+\infty} \exp \left[-(z / b)^{2}-z-\frac{t}{\tau_{\max }} e^{-z}\right] \mathrm{d} z,
\end{aligned}
$$

die bei JAhnкE-Eмde ${ }^{4}$ tabelliert ist. Abb. 1 zeigt $\eta(t)$ in doppel-logarithmischer Darstellung mit $b$ als Parameter.

Nun erweitern wir den Ansatz - Verteilung der Relaxationszeiten um e in en häufigsten Wert $\tau_{\max }$ und nehmen mehrere Häufungspunkte $\tau_{\max }^{\mathrm{I}}, \tau_{\max }^{\mathrm{II}}, \ldots$ an. Die resultierende Nachwirkungsstromdichte

3 K. W. W Elektrotechn. Z. 34, 1280 [1913].

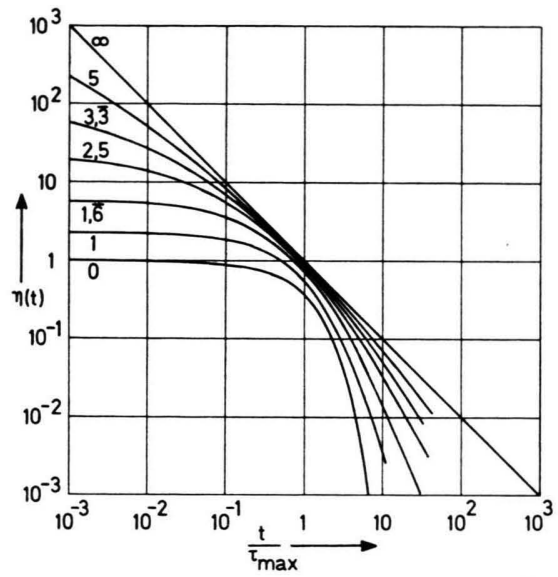

Abb. 1. Nachwirkungsfunktion $\eta(t)=j_{\text {normiert mit } b \text { als Para- }}$ meter.

$j_{\text {Gesamt }}$ erhalten wir zu

$$
j_{\text {Gesamt }}=j_{0}^{\mathrm{I}} j_{\text {normiert }}^{\mathrm{I}}+j_{0}^{\mathrm{II}} j_{\text {normiert }}^{\mathrm{II}}+\ldots ;
$$

$j_{0}^{\mathrm{I}}, j_{0}^{\mathrm{II}}, \ldots$ sind dabei Gewichtsfaktoren. Abb. 2 a, b, c zeigen das Resultat der Überlagerung für drei Spezialfälle in doppel-logarithmischer Darstellung,

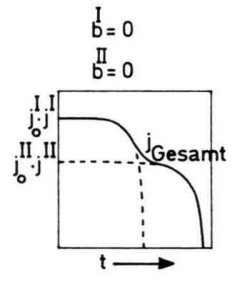

a

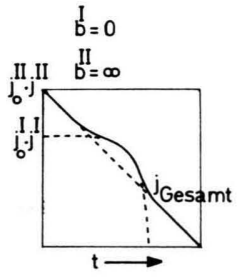

b

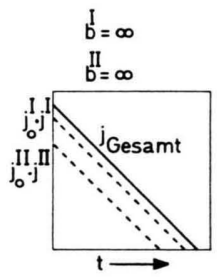

c
Abb. 2. Uberlagerung von jeweils zwei Nachwirkungsströmen.

wenn man nur zwei Häufungspunkte annimmt. In den Zeichnungen ist $\tau_{\max }^{\mathrm{I}}<\tau_{\max }^{\mathrm{II}}$ und außerdem $j_{0}^{\mathrm{I}}>j_{0}^{\mathrm{II}}$. Wäre $j_{0}^{\mathrm{I}}<j_{0}^{\mathrm{II}}$, so würde der Anteil $j_{0}^{\mathrm{II}} j_{\text {normiert zu allen }}^{\mathrm{II}}$ Zeiten den Anteil $j_{0}^{\mathrm{I}} j_{\text {normiert }}^{\mathrm{I}}$ überwiegen, und dieser Fall wäre wie der der Abb. $2 \mathrm{c}$ nicht von einem einzigen Häufungspunkt $\tau_{\max }$ zu unterscheiden.

\section{Anlage der Experimente}

Die meisten Experimente verliefen nach folgendem Schema. Die zu untersuchende $\mathrm{BaTiO}_{3}$-Probe lagerte einige Zeit bei der Meßtemperatur $T_{0}$. Dann folgten drei Meßabschnitte:

${ }^{4}$ E. Jahnke u. F. Emde, Funktionentafeln, Teubner-Verlag, 2. Aufl., Berlin-Leipzig 1933. 
1. Für die Zeit $\Delta_{1}$ lag eine Gleichspannung $U$ an der Probe, und der hierbei fließende Strom $J_{1}(t)$ wurde registriert.

2. Nach der Zeit $\Delta_{1}$ wurde die Probe über das Strommeßgerät kurzgeschlossen und der nun fließende Strom $J_{2}(t)$ aufgezeichnet.

3. Nach einer festen Zeit $\Delta_{2}$ oder wenn der Strom $J_{2}(t)$ auf einen bestimmten Wert abgeklungen war, wurde die Probe mit annähernd linearem $T(t)$-Anstieg erwärmt, die Endtemperatur war bei den einzelnen Messungen verschieden. Der beim Hochheizen von der weiter kurzgeschlossenen Probe fließende Strom $J_{3}(t)$ wurde ebenfalls meist registriert.

Die Ströme $J_{1}(t)$ und $J_{2}(t)$ werden immer in doppellogarithmischer Auftragung wiedergegeben. Sie können dann durch Translation stückweise mit $\eta(t)$ zur Deckung gebracht werden. Für die Messungen wurden grobkristalline Proben von Kreisscheibenform verwendet, die von der Firma $\mathrm{Stem}$ a freundlicherweise zur Verfügung gestellt worden waren. Die Daten der Proben und der eingebrannten Silberelektroden waren:

\begin{tabular}{|c|c|c|c|c|c|}
\hline Dicke & $\begin{array}{c}\text { Durch- } \\
\text { messer }\end{array}$ & $\begin{array}{c}\mid c \\
\text { durch- } \\
\text { messer }\end{array}$ & fläche & $\begin{array}{c}\text { Korn- } \\
\text { größe }\end{array}$ & Porosität \\
\hline $1 \mathrm{~mm}$ & $20 \mathrm{~mm}$ & $19 \mathrm{~mm}$ & $2,84 \mathrm{~mm}^{2}$ & $7-14 \mu$ & $2,3 \%$ \\
\hline
\end{tabular}

Die damit erreichten Meßergebnisse decken sich quantitativ, solange der Korngrößenbereich derselbe ist. Wird er variiert, so bleibt das Verhalten qualitativ dasselbe.

\section{Ergebnisse der $J_{1}(t)$-Messungen}

Abb. 3 zeigt den Verlauf des Stromes $J_{1}(t)$ bei der Spannung $U=370 \mathrm{~V}$, aber bei sechs verschiedenen Temperaturen. Nur bei $10^{\circ} \mathrm{C}$ sieht man den

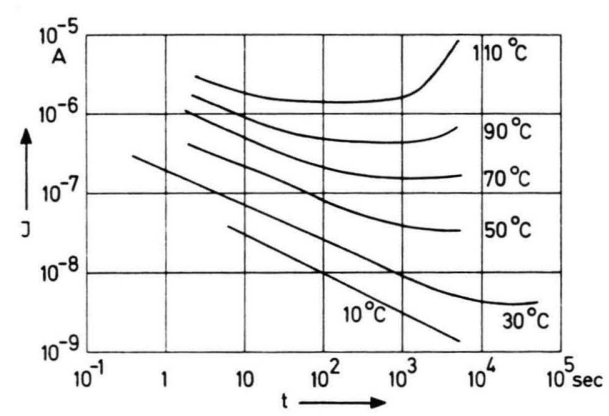

Abb. 3. $J_{1}(t)$ mit $U=370 \mathrm{~V}$ und $T_{0}$ als Parameter.

erwarteten Stromverlauf: Der Nachwirkungsstrom $J_{N}$ nimmt mit der Zeit ab, so daß sich der gemessene Gesamtstrom immer mehr dem Leitfähigkeitsstrom $J_{\mathrm{L}}$ nähert. Dieser wird allerdings nicht erreicht. Bei den

5 G. Busch, H. Flury u. W. Merz, Helv. Phys. Acta 21, 212 [1948]. höheren Temperaturen sinkt der Strom anfangs ebenfalls, strebt aber keinem konstanten Wert zu, sondern durchläuft ein Minimum und steigt dann wieder an. Das Minimum verschiebt sich mit wachsender Temperatur zu kürzeren Zeiten. Ebenfalls mit zunehmender Temperatur verschieben sich die ganzen Kurven nach oben zu größeren Stromwerten. Dies letzte ist verständlich, da die Leitfähigkeit - nach ${ }^{5-7}$ - und damit der Leitfähigkeitsstrom mit steigender Temperatur nach dem bekannten Gesetz

$$
\sigma=A e^{-W / k T}
$$

wachsen.

\section{Ergebnisse der $J_{2}(t)$-Messungen und ihre erste Interpretation}

\section{a) Einfluß der Ladezeit $\Delta_{1}$ und der Meßtemperatur $T_{0}$}

Die Abb. 4 zeigt den Verlauf des Stromes $J_{2}(t)$. Für jede der 4 Kurvenscharen ist jeweils die Temperatur der Probe fest. Dagegen ist die Zeit $\Delta_{1}$ bei den Kurven jeder Schar variiert. Die Spannung be. trug stets $U=370 \mathrm{~V}$. In Abb. 5 sind alle 5000 secMessungen (auch die bei 10 und $110^{\circ} \mathrm{C}$ ) zusammengefaßt.

Bei $30{ }^{\circ} \mathrm{C}$ wächst der Strom $J_{2}$ bei $t=$ const mit zunehmenden $\Delta_{1}$-Werten und strebt einer Grenzkurve zu. Bei den höheren Meßtemperaturen gilt dies nur bis gegen $1000 \mathrm{sec}$. Diese Grenzkurven werden von allen $\Delta_{1}=5000$ sec-Messungen, soweit man sich auf kleine Zeiten beschränkt, gut angenähert. Dieses Verhalten von $J_{2}(t)$ kann man mittels der in Abschnitt 1 dargestellten Modelle verstehen. Das allmähliche Erreichen der Grenzkurven mit wachsendem $\Delta_{1}$ weist auf einen umkehrbaren Relaxationsmechanismus und gleichzeitig auf eine Verteilung von Relaxationszeiten um einen häufigsten Wert hin. Er heiße $\tau_{\max }^{\mathrm{II}}$. Eine Grenzkurve erhalten wir nach diesen Modellvorstellungen erst dann, wenn für alle um $\tau_{\max }^{\mathrm{II}}$ gruppierten Relaxationszeiten $\tau$ die Bedingung für volle Anregung $\Delta_{1} \geqq 4,6 \tau$ erfüllt ist. Trifft dagegen diese Bedingung nur für einen Teil der Relaxationszeiten zu, so weicht die Meßkurve bei größeren Zeiten $t$, da Mechanismen mit zu großem $\tau$ nicht voll angeregt sind, von der Grenzkurve ab. Dieses Verhalten sieht man in allen vier Kurvenscharen der Abb. 4. Die Meßkurven mit $\Delta_{1}=500 \mathrm{sec}$ fallen mit den $\Delta_{1}=5000 \mathrm{sec}-$ Kurven bis $t=50 \mathrm{sec}$

${ }^{6}$ S. Nomura u. S. Sawade, J. Phys. Soc. Japan 5, 227 [1950].

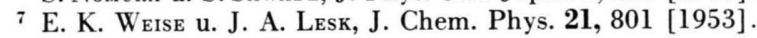




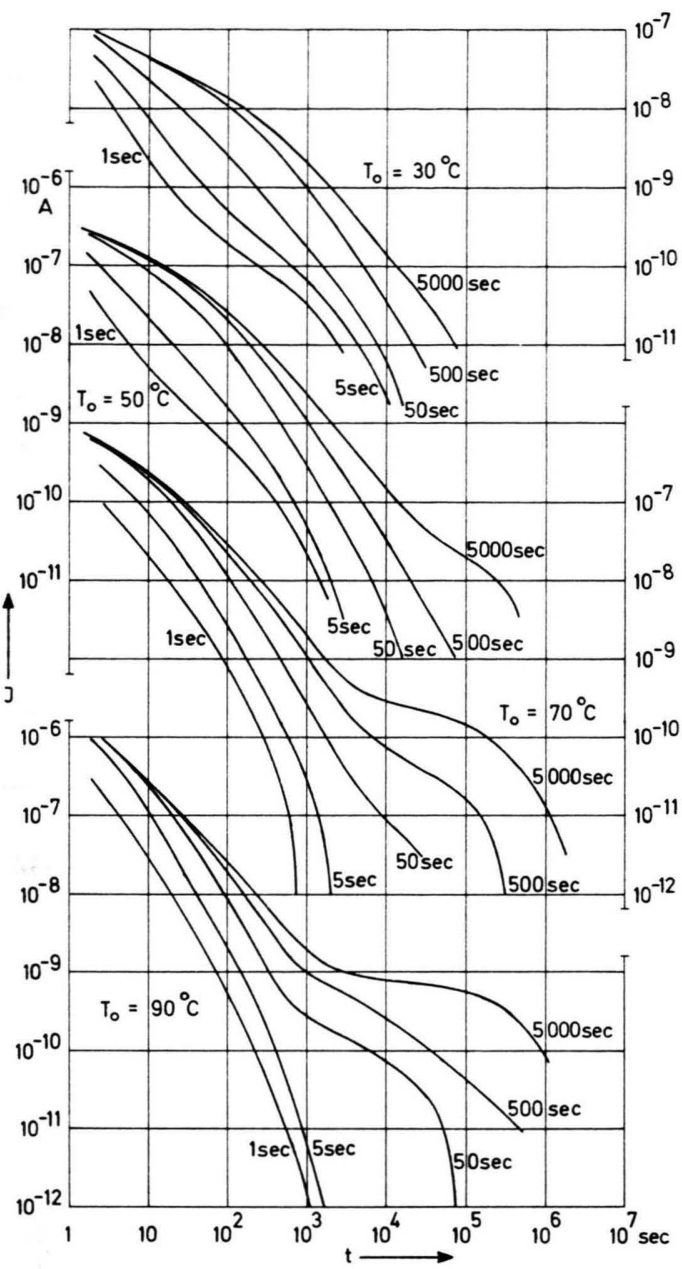

Abb. 4. $J_{2}(t)$ mit $U=370 \mathrm{~V}$ und $T_{0}$ und $\Delta_{1}$ als Parameter.

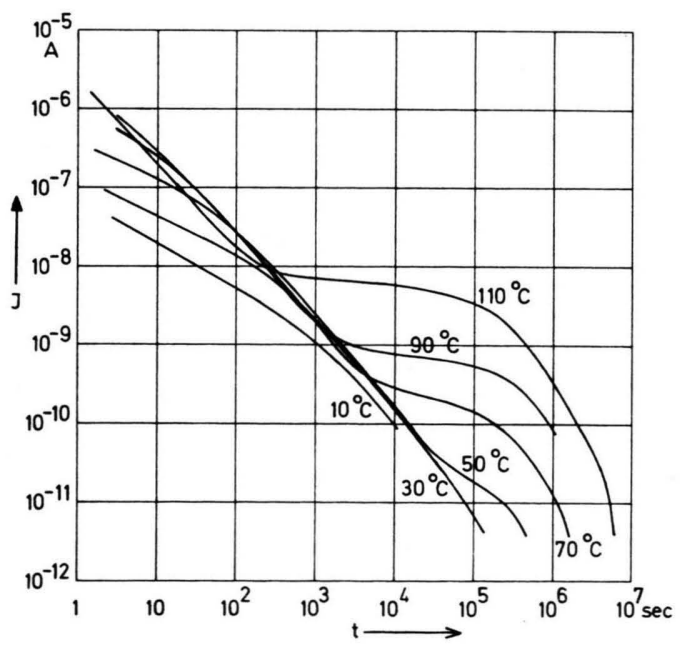

Abb. 5. $J_{2}(t)$ mit $\Delta_{1}=5000 \mathrm{sec}$ und $U=370 \mathrm{~V}$, Parameter ist $T_{0}$. zusammen, die Meßkurven mit $\Delta_{1}=50 \mathrm{sec}$ dagegen nur bis $t=2$ bis $10 \mathrm{sec}$. Die $\Delta_{1}=5000 \mathrm{sec}$-Messungen dürften also durch Extrapolation bis etwa $t=1000 \mathrm{sec}$ mit den Grenzkurven identisch sein.

Von dem beschriebenen einfachen Verhalten (Verteilung der Relaxationszeiten um nur einen häufigsten Wert und umkehrbarer Relaxationsmechanismus) weichen die Meßkurven $J_{2}(t)$ an zwei verschiedenen Stellen ab:

1. Man erwartet, daß die Steigung der Kurven $J_{2}(t)$ um so größere negative Werte annimmt, je kleiner die Anregungszeit $\Delta_{1}$ ist, wie man dies bei $T_{0}=70{ }^{\circ} \mathrm{C}$ links auch sehen kann. Bei $30{ }^{\circ} \mathrm{C}$ und auch bei $50{ }^{\circ} \mathrm{C}$ dagegen zeigt sich, daß die Steigung der gemessenen Kurven mit dem Parameterwert $\Delta_{1}=1 \mathrm{sec}$ und weniger deutlich auch bei $\Delta_{1}=5 \mathrm{sec}$ anfangs tatsächlich größere negative Werte besitzt, dann jedoch nimmt der Betrag der Steigung wieder ab. Erst danach werden die Kurven endgültig immer steiler. Zahlreiche weitere Messungen bei Zwischentemperaturen fügen sich diesem Verhalten vollständig ein. Das Bild erinnert sofort an die Abb. 2 a und damit an zwei Häufungspunkte $\tau_{\max }^{I}$ und $\tau_{\max }^{I I}$ der Relaxationszeiten. Daß die Hochwölbung, die zu der Relaxationszeit $\tau_{\max }^{\mathrm{I}}$ gehört, nur bei niedrigen Temperaturen und kurzen Anregungszeiten $\Delta_{1}$ sichtbar wird, liegt daran, daß ihr Gewichtsfaktor $j_{0}{ }^{\mathrm{I}}$ weniger mit der Temperatur abnimmt und ihr Mechanismus offenbar nicht umkehrbar ist. Dies werden wir erst im Zusammenhang mit anderen Messungen verstehen.

2. Bei höherer Meßtemperatur und größeren Zeiten $\Delta_{1}$ sieht man im Bereich $t>1000$ sec keine Grenzkurve, sondern die Meßkurven wölben sich ebenfalls auf. Dies ist sofort verständlich, wenn man eine Verteilung der Relaxationszeiten $\tau$ wieder um zwei häufigste Werte $\tau_{\max }^{\mathrm{II}}$ und $\tau_{\max }^{\mathrm{III}}$ annimmt, wobei der Mechanismus bei $\tau_{\max }^{\mathrm{III}}$ einen kleineren Gewichtsfaktor besitzt, der ebenfalls mit steigender Temperatur wächst. Um eine Grenzkurve im Bereich $t>1000 \mathrm{sec}$ zu erhalten, müßte man $\Delta_{1} \geqq 4,6 \tau_{\max }^{\mathrm{III}}=15$ Tage wählen. Diese großen Anregungszeiten $\Delta_{1}$ konnten leider nicht verwendet werden, da vor allem bei höheren Temperaturen die Proben vorher zerstört werden.

\section{b) Einfluß der Ladespannung}

Auch der Einfluß der zuvor angelegten Ladespannung auf $J_{2}(t)$ wurde untersucht. Das Ergebnis dieser Messungen für $T_{0}=30{ }^{\circ} \mathrm{C}$ zeigt zusammen- 
fassend die Abb. 6. In ihr sind in doppel-logarithmischer Darstellung die Stromwerte $J_{2}$ bei $t=10$, 100 und 1000 sec über der Spannung aufgezeichnet. Der Strom $J_{2}$ wächst hiernach nahezu linear mit der Spannung an.

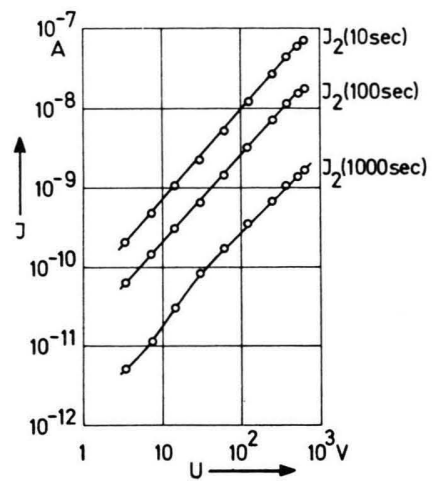

Abb. 6. $J_{2}(U)$ bei $T_{0}=30{ }^{\circ} \mathrm{C}$ und $\Delta_{1}=500 \mathrm{sec}$, Parameter ist die Meßzeit.

\section{c) Quantitative Auswertung der Kurven $J_{2}(t)$}

Man hat schließlich die Kurven $J_{2}(t)$ quantitativ mit der Nachwirkungsfunktion $\eta(t)$ zu vergleichen. Dabei ergeben sich Zahlenwerte für $J_{0}, \tau_{\max }$ und $b$, die zum Teil in Abb. 7 wiedergegeben sind. Auf der Abszisse ist der Kehrwert der absoluten Temperatur aufgetragen und auf der Ordinate in logarithmischer Teilung $\tau_{\max }^{\mathrm{II}}, \tau_{\max }^{\mathrm{III}}, J_{0}^{\mathrm{II}}=F_{\text {Elektrode }} j_{0}^{\mathrm{II}}$ und $J_{0}^{\mathrm{III}}=$ $F_{\text {Elektrode }} j_{0}^{\mathrm{III}}$. $J_{0}^{\mathrm{II}}$ und $J_{0}^{\mathrm{III}}$ sind die Gewichtsfaktoren der beiden Relaxationsverteilungen mit den längeren Zeiten; sie wachsen nach Abb. 7 exponentiell $\left(\sim e^{-E / k T_{0}}\right)$ mit steigender Temperatur. Die Relaxationsverteilung mit den kürzesten Zeiten um $\tau_{\max }^{\mathrm{I}}$

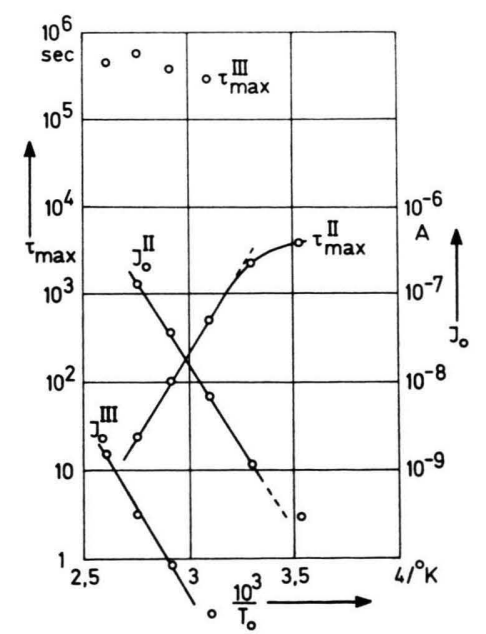

Abb. 7. $\tau_{\max }$ und $J_{0}$ als Funktion der Temperatur. kann nicht ausgewertet werden, da sie nur als Störung auftritt. Die Relaxationszeit $\tau_{\max }^{\mathrm{II}}$ sinkt exponentiell $\left(\sim e^{+E / k T_{0}}\right)$ mit steigender Temperatur; die erhebliche Abweichung bei $10^{\circ} \mathrm{C}\left(\triangleq 3,5 \cdot 10^{-3} /{ }^{\circ} \mathrm{K}\right)$ liegt daran, daß wir hier mit der $\Delta_{1}=5 \cdot 10^{3}$ secMessung die Grenzkurve gar nicht erreichen, da die Bedingung $\Delta_{1} \geqq 4,6 \tau$ für den überwiegenden Teil der Relaxationszeiten nicht erfüllt ist; diese Abweichung ist schon bei $30{ }^{\circ} \mathrm{C}\left(\triangleq 3,3 \cdot 10^{-3} /{ }^{\circ} \mathrm{K}\right)$ angedeutet. $\tau_{\max }^{\mathrm{III}}$ ist annähernd konstant $(3 \mathrm{bis} 6) \cdot 10^{5}$ sec, wenn auch (wie $J_{0}^{\mathrm{III}}$ ) unsicher wegen nicht erreichter Grenzkurve. Die Parameter $b$ sind bei den beiden ausgewerteten Vorgängen kaum von der Temperatur abhängig; sie betragen

$$
b^{\mathrm{II}}=3,3 \text { bis } 5 \text { und } b^{\mathrm{III}}=0 \text { bis } 1,
$$

d. h. die Relaxationszeiten sind sehr breit um $\tau_{\max }^{\mathrm{II}}$ gestreut, dagegen nur sehr wenig um $\tau_{\max }^{\mathrm{III}}$. Auf die Bedeutung dieser Ergebnisse werden wir später zurückkommen.

Um die bei der Relaxation transportierten Ladungen zu erhalten, wurden die Kurven der Abb. 5 numerisch integriert. Abb. 8 zeigt als Ergebnis den

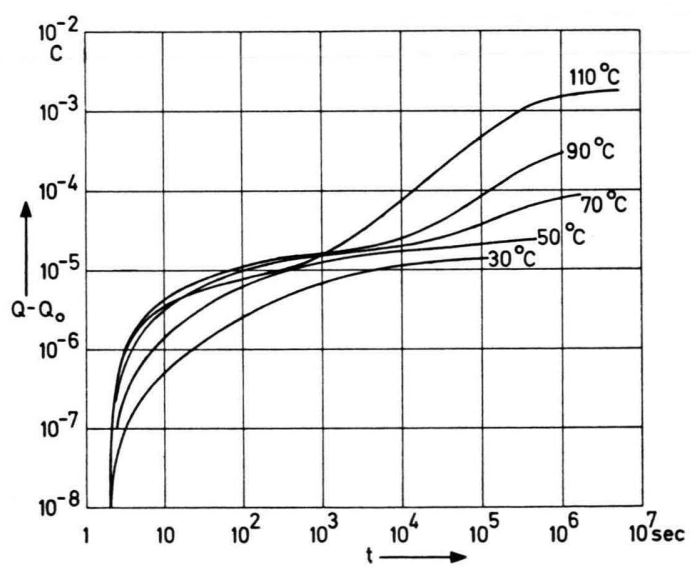

Abb. 8. $Q(t)-Q_{0}$ mit $\Delta_{1}=5000$ sec und $U=370 \mathrm{~V}$, Parameter ist $T_{0}$.

Wert des Integrals von $2 \mathrm{sec}$ an bis zum Zeitpunkt $t$, d. h. die bis $t$ transportierte Ladung $Q(t)$ abzüglich der bis $t=2 \mathrm{sec}$ transportierten Ladung $Q_{0}$. Im Diagramm sind Zeitachse (Abszisse) und Ordinatenachse logarithmisch geteilt. Auch an dieser Darstellung erkennt man deutlich die beiden verschiedenen Relaxationsmechanismen II und III. Man sieht, daß die Ladung einem Sättigungswert von etwa $20 \mu \mathrm{C}$ zustrebt (II), um dann $\left(T_{0}>30{ }^{\circ} \mathrm{C}\right)$ plötzlich wieder zuzunehmen und mit wachsender Temperatur immer größere neue Sättigungswerte zu erreichen. 


\section{Ergebnisse der $J_{3}(t)$-Messungen und ihre erste Auswertung}

a) Messungen bei variabler Ausgangstemperatur $T_{0}$

Bei den in Abb. 9 und 10 wiedergegebenen $J_{3^{-}}$Kurven war stets $\Delta_{1}=5 \cdot 10^{3} \mathrm{sec}$ und $\Delta_{2}=10^{4} \mathrm{sec}$. Parameter ist die Ausgangstemperatur $T_{0}$. Auf der Abszissenachse ist die Temperatur der Thermostatenflüssigkeit aufgetragen, die linear mit der Zeit wächst. Der Zusammenhang mit der Probentemperatur ist von Ernsthausen ${ }^{8}$ untersucht: Diese steigt zuerst

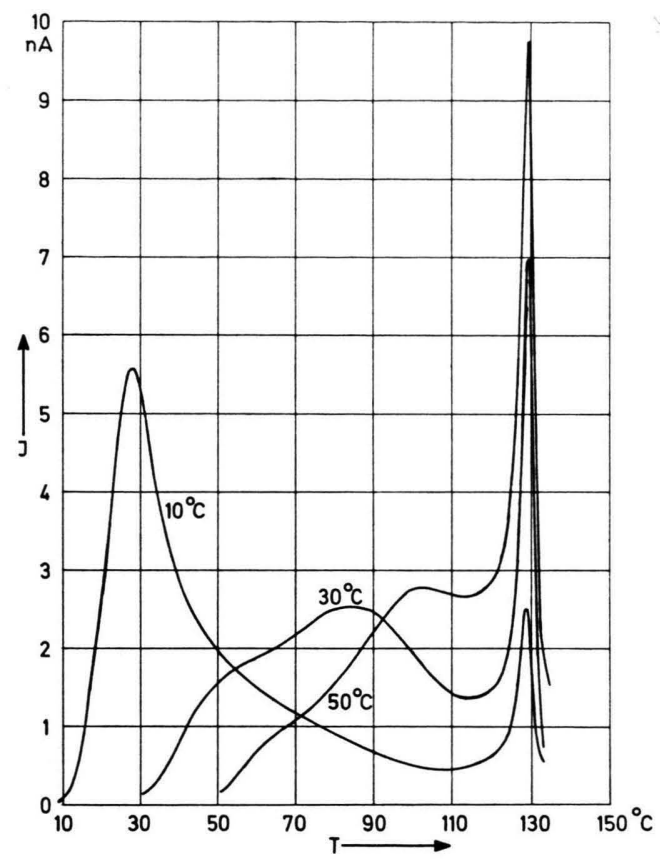

Abb. 9. $J_{3}(T)$ für $\Delta_{2}=10^{4} \mathrm{sec}$, Parameter ist $T_{0}$.

langsamer an als die Thermostatentemperatur, verläuft nach $\Delta T=8{ }^{\circ} \mathrm{C}$ parallel zu dieser, aber um etwa $10{ }^{\circ} \mathrm{C}$ zu kleineren Werten verschoben.

Alle fünf Meßkurven haben ein großes Maximum bei $130^{\circ} \mathrm{C}$ entsprechend einer Probentemperatur von $120{ }^{\circ} \mathrm{C}$, d. h. beim Curie-Punkt des ferroelektrischen Bariumtitanats. Es heiße „CurIe-Maximum“. Die Höhe dieses Maximums nimmt zuerst deutlich mit der Ausgangstemperatur zu, bleibt dann jedoch annähernd konstant.

Außer diesem Maximum beim CurIE-Punkt zeigen die Kurven, die zu den Ausgangstemperaturen $T_{0}=10,30$ und $50{ }^{\circ} \mathrm{C}$ gehören, noch weitere

8 J. Ernsthausen, Staatsexamensarbeit 1961, Institut für theoretische Physik der Technischen Hochschule Karlsruhe.

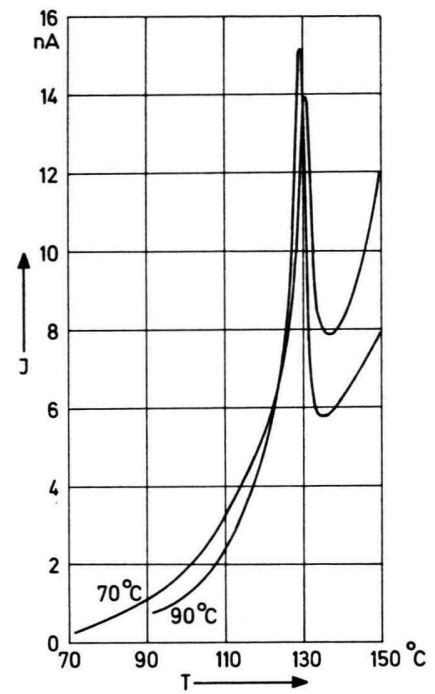

Abb. 10. $J_{3}(T)$ für $\Delta_{2}=10^{4} \mathrm{sec}$, Parameter ist $T_{0}$.

Maxima. Bei der Kurve mit $T_{0}=10^{\circ} \mathrm{C}$ liegt das besonders stark ausgeprägte Maximum bei etwa $28{ }^{\circ} \mathrm{C}$ (Probentemperatur $18^{\circ} \mathrm{C}$ ), d. h. gerade dort, wo die Zunahmegeschwindigkeit der Temperatur ihren größten Wert erreicht hat und dann konstant bleibt. Es heiße „erstes“ Maximum. Ein entsprechendes Maximum ist auch bei der Kurve mit $T_{0}=30{ }^{\circ} \mathrm{C}$ angedeutet, und zwar bei $T=50{ }^{\circ} \mathrm{C}$ entsprechend der Probentemperatur $40{ }^{\circ} \mathrm{C}$; es ist jedoch viel kleiner und daher von einem weiteren, größeren Maximum bei $T=85{ }^{\circ} \mathrm{C}$ (Probentemperatur $75^{\circ} \mathrm{C}$ ) - es heiße „mittleres“ Maximum - überragt, so daß es nur als Buckel auf dessen linker Flanke sichtbar ist. Ähnlich liegen die Verhältnisse bei der Kurve für $T_{0}=50{ }^{\circ} \mathrm{C}$ : Das erste Maximum ist noch kleiner geworden und kaum mehr als Buckel auf der linken Flanke des mittleren Maximums zu erkennen. Dieses selbst ist zu größerer Temperatur $T=103{ }^{\circ} \mathrm{C}$ (Probentemperatur $93{ }^{\circ} \mathrm{C}$ ) verschoben und kleiner geworden. Es liegt selbst wieder auf der linken Flanke des Curie-Maximums. Bei den Kurven mit den höheren Ausgangstemperaturen $\left(T_{0}=70\right.$ und $\left.90{ }^{\circ} \mathrm{C}\right)$ steigt der Strom $J_{3}(t)$ monoton und ohne Andeutung eines Buckels bis zum Curie-Maximum an, fällt dann ab, um aber schließlich doch wieder anzusteigen, vgl. Abschnitt 5 c.

\section{b) Messungen mit verschiedenen Zeiten $\Delta_{2}$}

Dabei war auch hier $\Delta_{1}=5 \cdot 10^{3} \mathrm{sec}$ und die Ausgangstemperatur $T_{0} 30^{\circ} \mathrm{C}$ in Abb. 11 und $50{ }^{\circ} \mathrm{C}$ in Abb. 12. Das Zeichen „(1)“ weist auf die entsprechenden Kurven der Abb. 9 mit $\Delta_{2}=10^{4} \mathrm{sec}$ hin. 


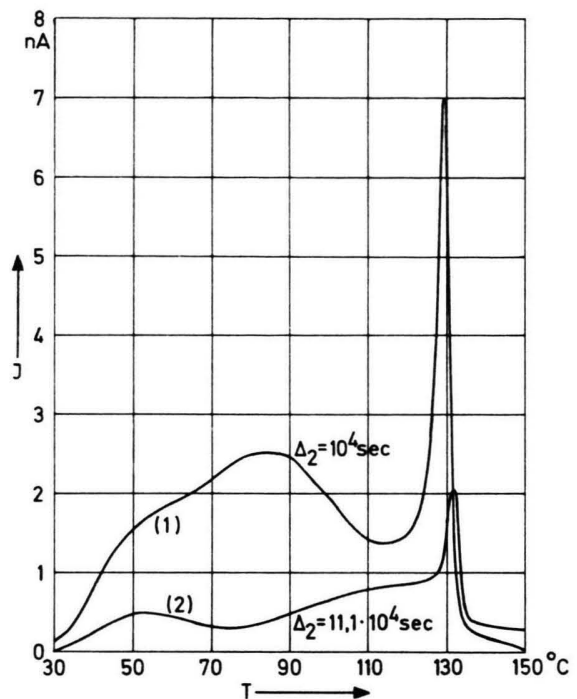

Abb. 11. $J_{3}(T)$ bei $T_{0}=30^{\circ} \mathrm{C}$, Parameter ist $\Delta_{2}$.

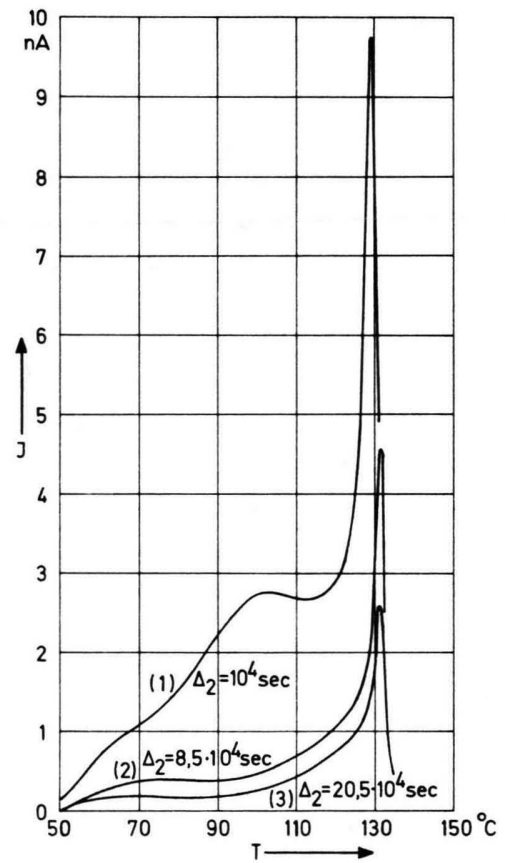

Abb. 12. $J_{3}(T)$ bei $T_{0}=50^{\circ} \mathrm{C}$, Parameter ist $\Delta_{2}$.

In beiden Diagrammen erkennt man, daß das CuRIEMaximum sehr stark mit wachsenden $\Delta_{2}$-Werten abnimmt, ebenso das "mittlere“ Maximum, das man in Abb. 12 bei den Kurven (2) und (3) gar nicht mehr erkennen kann und das in Abb. 11 bei (2) nur mehr als Hochwölbung auf der linken Flanke des CurieMaximums angedeutet ist. Dagegen ist das „erste“ Maximum bei größeren $\Delta_{2}$-Werten in Abb. 11 kaum kleiner geworden. In Abb. 12 ist es bei (2) nur noch schwach angedeutet.

Zum Vergleich wurden versuchsweise die durch $J_{2}$ und $J_{3}$ transportierten einander entsprechenden Ladungen berechnet, wobei jedoch fast nie Übereinstimmung auftrat. Dies hängt mit den benutzten oberen Integrationsgrenzen zusammen. Bei $J_{2}$ konnte immer nur bis zum letzten Meßwert integriert werden, während als Grenze $t=\infty$ sec richtig wäre. Bei $J_{3}$ wurde bis nach dem CurIE-Maximum integriert. Bei den weiteren Messungen zeigten sich aber jenseits $130{ }^{\circ} \mathrm{C}$ neue Erscheinungen, die zur Klärung zunächst studiert werden mußten.

\section{c) Weitere Messungen von $J_{3}(t)$ mit erweitertem Temperaturbereich}

Bei einem zweiten Ausheizen nach dem üblichen ersten findet man einen ebenfalls unerwarteten Strom $J_{3} \neq 0$. Er nimmt im wesentlichen monoton mit der Temperatur zu und hängt nicht von der Steilheit des Temperaturanstiegs ab. Dagegen wächst er mit zunehmender Gesamtzeit, während der überhaupt eine Spannung gleicher Polarität an der Probe lag.

Die Vermutung, daß wir etwa nur nicht genügend hochgeheizt haben, um Relaxationserscheinungen zu beseitigen, konnte widerlegt werden. Selbst mehrmaliges stundenlangse Hochheizen auf über $700{ }^{\circ} \mathrm{C}$ und monatelanges Lagern der Probe bei Zimmertemperatur änderte nichts an diesem nur von $T$ abhängigen Strom. Bei einer Reihe von Versuchen wurde dann das erste Hochheizen schon auf Temperaturen von 600 bis $700{ }^{\circ} \mathrm{C}$ ausgedehnt. Ein Beispiel zeigt Abb. 13. Beim Hochheizen von $110{ }^{\circ} \mathrm{C}$ an sehen wir zwei Maxima des Stromes $J_{3}$ : Das Curie-Maxi-

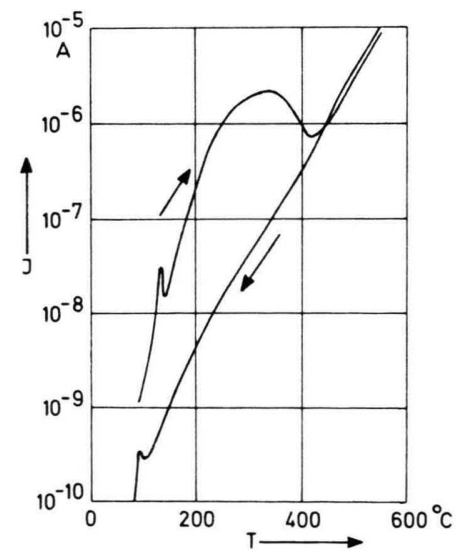

Abb. 13. $J_{3}(T)$ bei $T_{0}=110^{\circ} \mathrm{C}$. 
mum bei $140{ }^{\circ} \mathrm{C}$ und ein weiteres bei $340{ }^{\circ} \mathrm{C}$ (Anzeige des benachbarten Thermoelements). Dieses heiße künftig „viertes“ Maximum. Beim Abkühlen erhalten wir (bis auf ein kleines Maximum und Minimum beim Curie-Punkt) den anfangs erwähnten monotonen Stromverlauf, der reproduzierbar ist. Benutzt man niedrigere Ausgangstemperaturen, so liegen die beiden Kurven weniger weit auseinander. Voraussetzung ist, daß die Probe immer mit gleicher Polung an der Spannungsquelle lag. Anderenfalls erhält man ganz komplizierte Stromfunktionen, sogar mit Vorzeichenwechsel. Alle diese Ergebnisse deuten auf ein Diffusionsphänomen bei Anwesenheit eines elektrischen Feldes (vgl. Abschnitt $6 \mathrm{~d}$ ).

Trägt man die Stromkurven der Abb. 13 über 1/T auf, so entstehen beim Abkühlen stückweise Geraden wie bei den Leitfähigkeitskurven $\sigma(T)$. Man kann danach als Ursache unseres Stromes eine konstante Spannung vermuten, wobei seine Temperaturabhängigkeit allein durch die Leitfähigkeit $\sigma(T)$ verursacht würde. Rechnet man die notwendige Spannung aus, so erhält man $U=0,23 \mathrm{~V}$.

Aus der Abb. 13 wurde - bei Beachtung der Temperaturverschiebung beim Erwärmen und $\mathrm{Ab}$ kühlen der Probe - durch Differenzbildung der reine Relaxationsanteil des Stromes erhalten und die transportierte Ladung durch Integration ermittelt. Diese stimmt nun mit der entsprechenden aus $J_{2}$ gewonnenen Ladung (vgl. $5 \mathrm{~b}$ ) überraschend gut überein. Hiermit liegt die Vermutung nahe, daß der Ausheizbuckel bei $340^{\circ} \mathrm{C}$ mit der Relaxationserscheinung um $\tau_{\max }^{\mathrm{III}}$ zusammenhängt, die praktisch allein die von $J_{2}$ transportierte Ladung bestimmt (Abb. 8).

\section{Modelle zur Erklärung $\operatorname{der} J_{3}(t)$-Messungen}

\section{a) Deutung des Curie-Maximums}

Wenn man einen $\mathrm{BaTiO}_{3}$-Einkristall mit linear ansteigender Temperatur erwärmt, so fließt gemäß dem bekannten Verlauf der spontanen Polarisation ${ }^{9}$ ein immer größerer Strom, der beim CurIE-Punkt ein hohes Maximum erreicht und dann auf Null absinkt. Eine keramische Probe enthält viele einzelne Kristallite mit verschiedener Orientierung. Durch das während der Zeit $\Delta_{1}$ angelegte Feld ordnen diese ihre spontane Polarisation so um, daß die potentielle Energie zu einem Minimum wird, d. h. eine resul-

9 W. J. Merz, Phys. Rev. 91, 513 [1953]. tierende Polarisation in Feldrichtung entsteht. Nach dessen Abschalten wird die Vorzugsorientierung allmählich in den einzelnen Kristalliten teilweise abgebaut. Dieser Vorgang liefert während der Zeit $\Delta_{2}$ einen ferroelektrischen Beitrag zum Nachwirkungsstrom $J_{2}$.

Bei Beginn des Ausheizens ist dann noch eine gewisse Anzahl von Kristalliten übriggeblieben, deren spontane Polarisation bevorzugt orientiert ist. Setzt man vereinfachend voraus, daß keiner dieser Kristallite die Richtung seiner spontanen Polarisation während des Hochheizens ändert, so liefert dabei jeder einzelne einen Beitrag zum Ausheizstrom $J_{3}$, der ganz dem eines Einkristalles entspricht, d. h. einen Stromanteil, der monoton bis zum Curie-Punkt wächst, dort sein Maximum hat und dahinter Null wird.

In Wirklichkeit nimmt die Anzahl der orientierten Kristallite vom Beginn des Ausheizens an ständig ab und liefert dadurch schon unterhalb des Curie-Maximums weitere Beiträge zum Ausheizstrom $J_{3}$. Frühere pyroelektrische Messungen ${ }^{10}$ lassen vermuten, daß hierdurch ebenfalls ein Strommaximum zustande kommt. Es fragt sich, ob dies mit einem der früher als „erstes“ oder „mittleres" bezeichneten Maxima übereinstimmt (vgl. Abschn. 5 a) .

\section{b) Modell zur Temperaturabhängigkeit der Relaxationszeit}

Wir betrachten die dielektrische Verschiebung (vgl. Abschnitt 1) als Funktion der Zeit und der Temperatur. Bei unseren Hochheizversuchen ist auch die Temperatur eine Funktion der Zeit, also $D=D(t, T(t))$. Somit wird

$$
j=-\frac{\partial D}{\partial t}-\frac{\partial D}{\partial T} \cdot \frac{\mathrm{d} T}{\mathrm{~d} t} .
$$

Der erste Summand ist die - bei $T=$ const allein auftretende - schon bekannte Nachwirkungsstromdichte; der zweite Ausdruck der rechten Seite stellt eine zusätzliche „Ausheizstromdichte“ dar. Beim Ausheizen mißt man natürlich die Summe der beiden Terme.

Zur Anwendung dieser Beziehung wählen wir für $D(t, T)$ den gleichen einfachen exponentiellen Ansatz wie am Anfang von Abschnitt 1. Die Temperaturabhängigkeit von $D$ steckt nur in $\tau$. Sie laute - in

10 B. WILD, Diplomarbeit 1957, Institut für theoretische Physik der Technischen Hochschule Karlsruhe. 
Übereinstimmung mit theoretischen Modellen, z. B. ${ }^{11}$ - $\tau=A \cdot \exp [B / T]$. Wir haben uns damit gemäß Abb. 7 , wo nur $\tau_{\max }^{\mathrm{II}}$ exponentiell über $1 / T$ verläuft, auf diesen Relaxationsvorgang beschränkt und das folgende gilt daher auch nur für diesen. Damit wird die Stromdichte beim Ausheizen

$$
j=\frac{D_{0}}{\tau} e^{-t / \tau}\left(1+\frac{B t}{T^{2}} \cdot \frac{\mathrm{d} T}{\mathrm{~d} t}\right) .
$$

(Hierbei ist $D_{0}$ als Konstante behandelt, da sie nur von $T_{0}$, aber nicht von der variablen Ausheiztemperatur $T$ abhängt.) Wenn man hiermit den gemessenen Ausheizstrom darstellen will, hat man zunächst die gefundene Gleichung auf Extrema zu untersuchen. Die Rechnung führt auf die Lösung einer transzendenten Gleichung. Durch Näherung ergibt sich, daß nur ein Maximum existiert, das weit unterhalb des hier benutzten Temperaturbereiches liegt. In diesem selbst hat man nur eine monoton abfallende Ausheizstromdichte zu erwarten.

Für den Ablauf unserer Messungen sind Zeit, Temperaturen und nach der gefundenen Gleichung die erwarteten Stromdichten in Abb. 14 schematisch

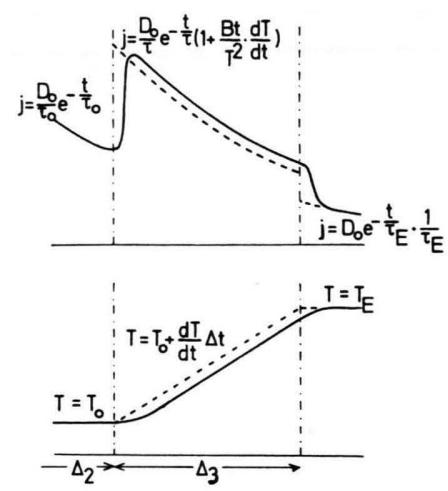

Abb. 14. Schema des Ausheizvorgangs.

dargestellt. Zur monoton fallenden Nachwirkungsstromdichte während $\Delta_{2}$ vor dem Ausheizen, die durch den Faktor vor der Klammer dargestellt wird, kommt während des Ausheizens $\left(\Delta_{3}\right)$ der zweite Summand in der Klammer hinzu. Nach dem Ausheizen, wenn eine neue, höhere Temperatur $T_{\mathrm{E}}$ erreicht ist, wird die Stromdichte wieder nur durch die Exponentialfunktion vor der Klammer, allerdings mit einem anderen $\tau_{\mathrm{E}}=\tau\left(T_{\mathrm{E}}\right)$ beschrieben. An Stelle

11 H. FröHLich, Theory of Dielectrics, Clarendon Press, 2. Aufl., Oxford 1958.

12 F. Jona u. G. Shirane, Ferroelectric Crystals, Pergamon Press, Oxford 1962. des theoretisch einfachen linearen Temperaturanstiegs im Ausheizbereich, der in Abb. 14 punktiert ist und Sprünge in $j$ hervorrufen würde, hat man in Wirklichkeit, wie in Abschnitt 5 a erläutert, den ausgezogenen Temperaturverlauf mit Abrundungen an beiden Enden. Dies bewirkt, daß $j$ statt eines Sprunges am Intervallanfang ein Maximum bei derjenigen Temperatur durchläuft, bei der die Neigung der $T(t)$-Kurve ihren konstanten Höchstwert erreicht. Diese Temperatur liegt nach Abschnitt 5 a um $8{ }^{\circ} \mathrm{C}$ oberhalb der Ausgangstemperatur $T_{0}$. Bei den Ausheizkurven von Abb. 9 liegt die Temperatur des „ersten“ Ausheizmaximums aber gerade ebenso viel über der Ausgangstemperatur. Damit erweist sich dieses als identisch mit dem hier theoretisch erhaltenen Maximum. Vorher wurde betont, daß die ganze Überlegung nur zu den Relaxationsvorgängen um $\tau_{\max }^{\mathrm{II}}$ gehört. Also ist das „erste“ Ausheizmaximum auch eindeutig mit $\tau_{\max }^{\text {II }}$ verknüpft.

Sucht man nun nach dem physikalischen Hintergrund für die Relaxationsvorgänge um $\tau_{\max }^{\mathrm{II}}$, so wird man auf die schon Maxwell bekannte Erscheinung geführt: Grenzen in einem Kondensator senkrecht zur Feldstärke zwei Gebiete aneinander mit unterschiedlichem Verhältnis $\varepsilon / \sigma$, so wird während $\Delta_{1}$ an diesen Grenzflächen Ladung gespeichert, die während $\Delta_{2}$ ebenso verzögert wieder abfließt, also einen Beitrag zum Nachwirkungsstrom $J_{2}(t)$ liefert. Nun wird jeder $\mathrm{BaTiO}_{3}$-Kristall bekanntlich ${ }^{12}$ von einer gegenüber dem Innern veränderten Randschicht von etwa 100 ÅE Dicke umgeben. Hierauf können wir die Ergebnisse für ein Modell aus zwei Schichten ${ }^{13}$ anwenden. Für die Relaxationszeit gilt unter einfachen Voraussetzungen

$$
\tau_{\text {Modell }} \sim 1 / \sigma
$$

Da aber nach ${ }^{5-7} \sigma$ proportional $e^{-K_{1} / T}$ ist, wird auch

$$
\tau_{\text {Modell }} \sim e^{+K_{1} / T} .
$$

Dieses theoretische Ergebnis ist in guter Utbereinstimmung mit dem experimentellen Befund (Abb. 11)

$$
\tau_{\max }^{\mathrm{II}} \sim e^{+K_{2} / T},
$$

wobei zudem der Wert der Konstanten $K_{2}$ im Streubereich der aus ${ }^{5-7}$ folgenden Werte von $K_{1}$ liegt. Diese hier nicht ganz zwingende Deutung des Relaxa-

13 W. F. Brown JR., Handbuch der Physik, Bd. 17, „Dielectrics“, Herausg. S. F FüGGE, Springer-Verlag, Berlin-Göttingen-Heidelberg 1956. 
tionsvorgangs um $\tau_{\max }^{\mathrm{II}}$ durch Ladungsansammlungen an derartigen Grenzschichten findet in den folgenden Abschnitten $6 \mathrm{~d}$ und 7 a weitere Bestätigung.

\section{c) Deutung des "mittleren“ Maximums}

Mit dem im letzten Abschnitt erhaltenen Ergebnis - Verknüpfung des „ersten“ Ausheizmaximums mit der Nachwirkungserscheinung um $\tau_{\max }^{\text {II }}$ - läßt sich gleichzeitig auf die am Ende von Abschnitt 6 a gestellte Frage durch Ausschluß antworten, daß das durch Herauskippen von Bereichen aus ihrer bevorzugten Orientierung während des Hochheizens erwartete Maximum mit dem „mittleren“ Ausheizmaximum identisch sein wird. Diese Deutung wird durch mehrere Tatsachen unterstützt:

1. Die starke Abnahme des „mittleren“ Maximums mit wachsenden Zeiten $\Delta_{2}$ (Abb. 11 und 12), d. h. wenn man genügend lange wartet, werden die zur Zerstörung der Vorzugsorientierung nötigen höheren thermischen Energien auch bei niedrigeren Temperaturen geliefert.

2. Die Verschiebung der Lage des „mittleren“ Maximums zu höheren Temperaturen und die rasche Abnahme seiner Höhe mit wachsender Ausgangstemperatur (Abb. 9 und 10). Bei höherer Ausgangstemperatur wird schon vor dem Beginn des Ausheizens in mehr Kristalliten mit bevorzugter Orientierung des Polarisationsvektors die thermische Energie zur Desorientierung ausreichen und so die Höhe des Maximums verringern. Vor allem die Kristallite, die eine kleine Aktivierungsenergie benötigen, werden vorzeitig desorientiert und bewirken so eine Verschiebung des „mittleren“ Maximums zu höheren Temperaturen.

3. Bei der Ausgangstemperatur von $10{ }^{\circ} \mathrm{C}$ ist das Curie-Maximum relativ klein und das "mittlere“ Maximum kaum angedeutet. Bei dieser Temperatur reichte nämlich das vorher während der Zeit $\Delta_{1}$ angelegte Feld nur zu schwacher Vorzugsorientierung der spontanen Polarisation in den Kristalliten. Also stehen auch für die folgende Zerstörung beim Ausheizen nur wenige Kristallite zur Verfügung.

\section{d) Deutung des „vierten“ Ausheizmaximums}

Dieses Maximum bei $T=340{ }^{\circ} \mathrm{C}$ (Abb. 13) hängt, wie wir schon im Abschnitt $5 \mathrm{c}$ feststellten, mit der

14 A. Branwood u. R. H. Tredgold, Proc. Phys. Soc. London 76, 93 [1960].
Relaxationserscheinung um $\tau_{\max }^{\mathrm{III}}$ zusammen. Zu seiner Deutung führen folgende schon bekannten experimentellen Ergebnisse:

1. Bei der Beschreibung des Stromes $J_{1}(t)$ (Abb. 3) wurde der erwartete konstante Leitfähigkeitsstrom außer bei der Messung bei $T_{0}=10^{\circ} \mathrm{C}$ nicht asymptotisch erreicht, sondern $J_{1}$ durchlief ein Minimum und stieg dann wieder an.

2. Bei den Ausheizversuchen trat mit steigender Temperatur neben den vier Maxima ein mit der Temperatur wachsender Strom auf, der unverändert bestehen blieb, auch nach mehrmaligem Ausheizen auf über $700{ }^{\circ} \mathrm{C}$.

Die Erscheinung 1. deutet auf allmähliche $\mathrm{Zu}$ nahme der Leitfähigkeit der Probe, 2. zudem auf die Ausbildung eines galvanischen Elementes. Beides kann dadurch entstehen, daß Elektrodenatome unter Feld einseitig in die Probe einwandern. Diese Vermutung wird durch Ergebnisse einiger Arbeiten ${ }^{14-16}$ an $\mathrm{BaTiO}_{3}$-Einkristallen bestätigt. Hiernach zeigen $\mathrm{BaTiO}_{3}$-Kristalle mit Silber- und Goldelektroden beim Anlegen großer Feldstärken (einige $\mathrm{kV} / \mathrm{cm}$ ) nach einiger Zeit (Stunden bis Tage) einen wachsenden Leitfähigkeitsstrom. Untersuchungen ${ }^{15}$ mit radioaktiven Goldelektroden ergaben, daß dabei Goldatome aus der Kathode in eine etwa $100 \AA \mathrm{E}$ dicke Schicht des $\mathrm{BaTiO}_{3}$-Einkristalls diffundieren und zur Bildung einer neuen Oberflächenschicht mit veränderten Eigenschaften führen.

Die obige Erscheinung 1. leuchtet damit ohne weiteres ein. Insbesondere wird die Diffusion bei höheren Temperaturen rascher verlaufen. - Die Erscheinung 2. deutet darauf hin, daß das asymmetrische Gebilde, das durch einseitige Diffusion von Silber aus der Kathode entsteht, galvanische Spannungen entwickeln kann.

Der Diffusionsvorgang ermöglicht nun aber auch, das "vierte" Ausheizmaximum zu verstehen. Die Silberatome der Kathode wandern durch Oberflächendiffusion (schneller als Diffusion ins Kristallinnere) zunächst über die Oberfläche der Kristallite und von da aus in deren Inneres. Dabei entsteht unsymmetrisch in den von diesem Vorgang erfaßten Kristalliten eine neue Grenzschicht, deren Eigenschaften gegenüber denen der in Abschnitt $6 \mathrm{~b}$ dargestellten normalen Kristallitrandzonen verändert sind und die daher Ursache für eine neue Relaxationserscheinung

15 A. Branwood, O. H. Hughes, J. D. Hurd u. R. H. Tredgold, Proc. Phys. Soc. London 79, 1161 [1962].

16 G. G. Roberts u. R. H. Tredgold, Phys. Letters 2, 6 [1962]. 
mit von $\tau_{\max }^{\mathrm{II}}$ verschiedener Relaxationszeit wird. Diesen Vorgang dürfen wir offensichtlich mit dem einzigen noch ungeklärten Nachwirkungsmechanismus $\tau_{\max }^{\text {III }}$ identifizieren. So wird schließlich auch das "vierte“ Ausheizmaximum, das mit $\tau_{\max }^{\mathrm{III}}$ zusammenhängt, verständlich.

Diese neuen Erkenntnisse bekräftigen zugleich die in Abschnitt $6 \mathrm{~b}$ vorgenommene Deutung von $\tau_{\max }^{\mathrm{II}}$. BullisGER ${ }^{17}$ denkt hierzu an die Grenzschicht Elektrodenmetall $/ \mathrm{BaTiO}_{3}$-Halbleiter. Wäre dies richtig, so müßte durch die Diffusion des Elektrodenmetalls bei höheren Temperaturen diese Grenzschicht wesentlich verändert und die Relaxationserscheinung um $\tau_{\max }^{\mathrm{II}}$ und damit $J_{2}(t)$ verringert werden. Davon findet sich in den Messungen aber keine Andeutung (Abb. 7). Deshalb ist unsere Annahme von Abschnitt $6 \mathrm{~b}$ richtig, die sich auf sämtliche inneren Korngrenzen der Keramik bezieht.

\section{Vergleich mit anderen Messungen}

\section{a) Messungen an feinkristallinen Proben}

Ernsthausen ${ }^{8}$ hat parallel zu dieser Arbeit entsprechende Messungen an keramischen $\mathrm{BaTiO}_{3}$. Proben mit Korngrößen kleiner als $1 \mu$ durchgeführt. Seine Ergebnisse über den Nachwirkungsstrom $J_{2}(t)$ zeigen nur den $\tau_{\max }^{\mathrm{II}}$ entsprechenden Relaxationsbuckel - allerdings mit viel größerem $b^{\text {II }}$. Auch bei den Ausheizkurven erkennt man ganz wesentliche Unterschiede. Nur das Curie-Maximum und das „erste“ Ausheizmaximum - ebenfalls immer $8{ }^{\circ} \mathrm{C}$ oberhalb der Ausgangstemperatur - treten überhaupt auf. Der Zusammenhang des „ersten“ Maximums mit der Relaxationserscheinung um $\tau_{\max }^{\mathrm{II}}$, der aus unserem Modell in Abschnitt $6 \mathrm{~b}$ folgt, ist also auch hier richtig. Jedoch fällt ein Unterschied beim Vergleich entsprechender Meßkurven sofort auf. Das Curie-Maximum ist bei den feinkristallinen Proben relativ zum ersten Maximum viel kleiner, d. h. das ferroelektrische Verhalten ist relativ viel schwächer ausgeprägt.

Dies ist sofort verständlich, wenn man das Verhältnis von gestörtem Randvolumen ${ }^{12}$ zum ungestörten Innenvolumen in Abhängigkeit von der Korngröße beachtet. Bei den feinkristallinen Proben überschreitet das Randvolumen $10 \%$ des Gesamtvolumens und damit werden alle rein ferroelektrischen Erscheinungen, die nur vom Innenvolumen abhängen, relativ schwach, so auch das Curie-Maximum. Dagegen müssen alle Effekte, die mit Grenzschichten an der Oberfläche der einzelnen Kristallite zusammenhängen, relativ ausgeprägt sein. So

17 G. Bullinger, Z. Angew. Phys. 12, 410 [1960]. verhält sich aber gerade das „erste“ Ausheizmaximum von Ernsthausen. Dieser Zusammenhang bekräftigt also noch einmal die Richtigkeit der in Abschnitt $6 \mathrm{~b}$ entwickelten Deutung dieses Maximums und des damit verbundenen Relaxationsmechanismus um $\tau_{\max }$.

\section{b) Vergleich mit den Messungen von Bullinger}

Bullinger ${ }^{17}$ hat bei Messung allein des Stromes $J_{2}(t)$ von $10^{-6}$ bis $100 \mathrm{sec}$ zwei Nachwirkungsmechanismen gefunden. Den ersten bezeichnet er als „Kurzzeitnachwirkung“ mit ferroelektrischer Ursache. Er ist mit dem bei uns nur angedeuteten Mechanismus um $\tau_{\max }^{\mathrm{I}}$ identisch. Zweitens ergab sich eine "Langzeitnachwirkung“, die bei den benutzten verschiedenen Proben nur qualitativ ähnliche Ergebnisse lieferte. Sie stimmt wohl mit unserem Relaxationsmechanismus um $\tau_{\max }^{\mathrm{II}}$ überein, obgleich außer erheblichen quantitativen Abweichungen auch qualitative Widersprüche existieren. Ein genauer Vergleich ist nicht sinnvoll, da vor allem im Gegensatz zu unseren möglichst reinen $\mathrm{BaTiO}_{3}$-Keramiken die von Bullinger verwandten Proben verschiedene erhebliche Beimengungen aufweisen.

\section{Gesamtübersicht über das Nachwirkungsverhalten}

Die ganzen Erscheinungen sind zunächst in Abb. 15 schematisch geordnet.

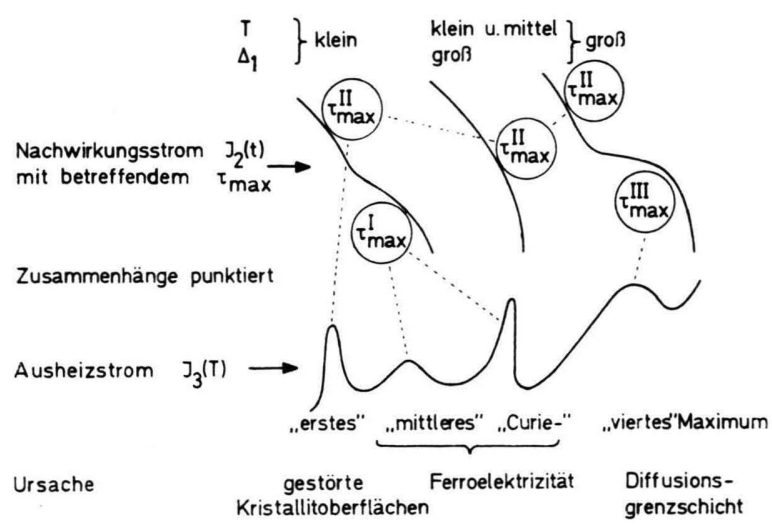

Abb. 15. Schema zum Zusammenhang der Erscheinungen.

a) Im einzelnen haben die rein ferroelektrische Nachwirkung vor allem Bullinger ${ }^{17}$ und KLEEFELD ${ }^{18}$ mittels der Nachwirkungsströme untersucht. Dabei ergaben sich Relaxationszeiten, unter denen die

18 C. Kleefeld, Diplomarbeit 1963, Institut für theoretische Physik der Technischen Hochschule Karlsruhe. 
kürzesten überhaupt vorkommenden Werte enthalten sind, die aber im ganzen besonders weit um ihr Mittel $\tau_{\max }^{\mathrm{I}}$ streuen. Die Ausläufer des Nachwirkungsstromes zu langen Zeiten finden sich auch in unseren Messungen. In Abb. 4 sieht man sie - dort als Anomalie hervorgehoben - mit Gewichtsfaktoren $J_{0} \mathrm{I}$, die wenig von der Temperatur abhängen, und vor allem mit einem Mechanismus, der im Sinn von Abschnitt 1 nicht umkehrbar ist. Beides hat man bei der rein ferroelektrischen Nachwirkung gerade zu erwarten: Der Gewichtsfaktor ist gegeben durch das Produkt (spontane Polarisation $X$ orientiertes Volumen). Beide Faktoren sind tatsächlich nur schwach temperaturabhängig verglichen mit dem exponentiellen Verhalten der Größe $J_{0}^{\mathrm{II}}$ (Abb. 7). Daß der Mechanismus nicht umkehrbar ist, ist ebenfalls verständlich: Die elektrische potentielle Energie spielt bei der Orientierung unter Feld eine größere Rolle als bei der Desorientierung nach Wegnahme des äußeren Feldes. Der erste Vorgang findet daher schneller statt als der zweite.

Auch im Ausheizstrom ist die ferroelektrische Nachwirkung nachweisbar, erstens in der Abnahme des Curie-Maximums mit wachsenden Zeiten (bis über $10^{5} \mathrm{sec}$ sichtbar) und zweitens in der noch rascheren Abnahme des mittleren Maximums mit wachsendem $\Delta_{2}$ (Abb. 11 und 12). Beides zeigt besser als die Messung des Nachwirkungsstromes - , daß die rein ferroelektrische Relaxation im „Untergrund" bis zu sehr langen Zeiten wirkt. Dies rechtfertigt auch die Annahmen von $\mathrm{KocH}^{1}$ zur Deutung seiner Messungen.

b) Die zweite Nachwirkungserscheinung, deren Relaxationszeiten um $\tau_{\max }^{\mathrm{II}}$ verteilt sind, findet man ebenfalls sowohl bei den grobkristallinen als auch bei den feinkristallinen Proben. Die hierbei auftretenden Verschiedenheiten (Abschnitt 7 a) weisen eindeutig auf die Grenzschichten der einzelnen Kristallite als Ursache der Erscheinung hin. Die daraus folgende Temperaturabhängigkeit von $\tau_{\max }^{\mathrm{II}}$ stimmt mit der gemessenen überein und ist dieselbe, die auch der Ausgangspunkt für das rein phänomenologische Modell von Abschnitt 6 b zur Deutung des ersten Ausheizmaximums war.

c) Den Nachwirkungsmechanismus mit der längsten Relaxationszeit $\tau_{\max }^{\mathrm{III}}$ und das zugehörige vierte Ausheizmaximum findet man nur bei den grobkristallinen Proben. Die Vorgänge werden allem Anschein nach durch eine Grenzschicht verursacht, die beim Diffundieren des Elektrodenmaterials in die $\mathrm{BaTiO}_{3}$-Keramik entsteht. Hiermit hängen zwei weitere Effekte zusammen, das Ansteigen des Leitfähigkeitsanteils von $J_{1}$ bei großen Temperaturen und großen Feldstärken und der Stromuntergrund beim Hochheizen.

Die Verteilung der Relaxationszeiten sieht nach allen Erfahrungen so aus: Bei kleinen Zeiten findet man nur ferroelektrische Relaxationszeiten, die so breit verteilt sind, daß eine Bestimmung von $\tau_{\max }^{\mathrm{I}}$ nicht möglich ist. Dann folgen die weniger breit verteilten Relaxationszeiten um $\tau_{\max }^{\mathrm{II}}$ und schließlich diejenigen eng um $\tau_{\max }^{\mathrm{III}}$. Die Lage der Maxima ist temperaturabhängig. Für $T$ zwischen 10 und $110^{\circ} \mathrm{C}$ bewegt sich $\tau_{\max }^{\mathrm{II}}$ von etwa $10^{4}$ bis $10, \tau_{\max }^{\mathrm{III}}$ von $2 \cdot 10^{5}$ bis $5 \cdot 10^{5} \mathrm{sec}$ (in dieser Reihenfolge).

Herrn Professor Dr. F. Wolf danke ich aufrichtig für Ratschläge und Unterstützung aller Art. Die Firma S t e a t it - M a g nesia AG., Lauf (Pegnitz), stellte dankenswerterweise die keramischen Proben zur Verfügung. 